\title{
Myxoinflammatory fibroblastic sarcoma: investigations by comparative genomic hybridization of two cases and review of the literature
}

\author{
Daniel Baumhoer • Kathrin Glatz • \\ Hans-Jürgen Schulten • László Füzesi • \\ Renato Fricker • Christoph Kettelhack • \\ Paula Hasenboehler • Martin Oberholzer • \\ Gernot Jundt
}

Received: 1 July 2007 / Accepted: 16 July 2007 / Published online: 11 August 2007

(C) Springer-Verlag 2007

\begin{abstract}
Myxoinflammatory fibroblastic sarcoma (MIFS) is a rare low-grade sarcoma of the distal extremities characterized by a myxohyaline stroma, a dense inflammatory infiltrate and virocyte- and lipoblast-like giant cells. Up to now, only two cases have been investigated cytogenetically, showing complex and heterogeneous karyotypes, in part with supernumerary ring chromosomes. We characterized two further cases of MIFS immunohistochemically and performed comparative genomic hybridization as well as DNA image cytometry analyses. Both tumors showed the
\end{abstract}

D. Baumhoer $(\bowtie) \cdot \mathrm{K}$. Glatz $\cdot$ M. Oberholzer $\cdot$ G. Jundt Institute of Pathology, University of Basel,

Schönbeinstrasse 40,

4003 Basel, Switzerland

e-mail: dbaumhoer@mac.com

G. Jundt

Bone Tumor Reference Center at the Institute of Pathology, University of Basel,

4003 Basel, Switzerland

H.-J. Schulten • L. Füzesi

Department of Pathology, University of Göttingen,

37099 Göttingen, Germany

R. Fricker

Department of Surgery, Kantonsspital Bruderholz,

4101 Bruderholz, Switzerland

C. Kettelhack

Department of Surgery, University of Basel,

4003 Basel, Switzerland

P. Hasenboehler

Department of Surgery, Bethesda Spital,

4020 Basel, Switzerland characteristic histomorphological pattern of MIFS and were positive for Vimentin and CD68. Moreover, both cases presented aberrant karyotypes including distinct DNA copy number changes involving chromosome 7 and disclosed DNA aneuploidy.

Keywords Myxoinflammatory fibroblastic sarcoma . Comparative genomic hybridization $\cdot \mathrm{CGH}$.

DNA image cytometry $\cdot$ Review

\section{Introduction}

In 1998, Montgomery et al. [14] first described inflammatory myxohyaline tumors of the distal extremities with virocyte or Reed-Sternberg-like cells as a distinct tumor entity with unique histologic features. In the same year, Meis-Kindblom et al. coined the shorter term acral myxoinflammatory fibroblastic sarcoma (MIFS) for the same lesion, usually presenting as poorly circumscribed multinodular mass of the distal extremities ranging from less than 1 to $8 \mathrm{~cm}$ in size $[2,12]$. Patients typically report a history of slowly growing and painless tumors that only occasionally are associated with pain or decreased mobility [2]. Histologically, MIFS exhibit a dense inflammatory infiltrate, predominantly consisting of lymphocytes and plasma cells, embedded in an alternating myxoid and hyaline stroma. Three main types of neoplastic cells can be distinguished, namely spindle-shaped cells, bizarre virocyte, or ganglion-like cells with enlarged nuclei and often huge, inclusion-like nucleoli and multinucleated lipoblast-like cells of variable size. Tumor cells are 
dispersed through the myxohyaline stroma as single cells or form coherent nodules $[2,9,12-14,17,18]$. The rates of local recurrences range between 22 and $67 \%$, probably depending on differences in surgical treatment, whereas distant metastases are exceedingly rare $(<2 \%$ of all reported cases) $[2,12,14,17]$.

Cytogenetic data on chromosomal aberrations of MIFS are scarce, and up to now, only two cases have been investigated so far. One of them revealed a complex karyotype with a reciprocal translocation $\mathrm{t}(1 ; 10)(\mathrm{p} 22 ; \mathrm{q} 24)$ in addition to the loss of chromosome 3 and 13, while the other exhibited supernumerary ring chromosomes and a derivate chromosome 13 with additional material on the short arm $[9,11]$. In this paper, we report two further cases of MIFS investigated by immunohistochemistry, comparative genomic hybridization (CGH), and DNA image cytometry.

\section{Materials and methods}

\section{Immunohistochemistry}

The biopsy specimens were formalin-fixed, routinely processed, paraffin-embedded, and stained with hematoxylin and eosin. The panel of applied primary antibodies, dilutions, and different methods of tissue preparation and pretreatment, respectively, are listed in Table 1 . All primary antibodies were diluted in a $1 \%$ solution of bovine serum albumin in phosphate-buffered saline $(\mathrm{pH}$ 7.4) and incubated for $30 \mathrm{~min}$ at room temperature. After incubation with the biotinylated secondary antibody, the avidinbiotin-peroxidase complex was applied for $30 \mathrm{~min}$, and color development was performed using diamino-benzidine
(DAB). The slides were counterstained with hemalaun, dehydrated, and mounted.

\section{Comparative genomic hybridization}

For CGH, tumor DNA was isolated from formalin-fixed and deparaffinized tumor tissue by proteinase $\mathrm{K}$ digestion $(2 \mathrm{mg} / \mathrm{ml}$ final concentration; Roche, Mannheim, Germany) followed by spin column purification (Qiagen, Hilden, Germany). Labeling of tumor DNA with biotin-16-dUTP (Roche, Mannheim, Germany) and normal reference DNA with digoxigenin11-dUTP (Roche) was performed by standard nick translation. The denatured DNA probe containing $2 \mu \mathrm{g}$ of tumor DNA, $1.5 \mu \mathrm{g}$ of reference DNA, and $80 \mu \mathrm{g}$ of COT-1 DNA was hybridized for 3 days to normal metaphase spreads on glass slides $(15 \times 15 \mathrm{~mm}$ cover glass area). The slides were then washed, blocked with bovine serum albumin solution, and incubated with fluorescein-conjugated avidin (Vector Laboratories, Burlingame, CA) and rhodamine-conjugated antidigoxigenin (Roche). Finally, the slides were washed and mounted in antifade solution (Vector Laboratories) containing $2.5 \mu \mathrm{g} / \mathrm{ml}$ of 4,6-diamino-2-phenylindole (DAPI) counterstain. Image acquisition was performed on a Zeiss Axioskop fluorescence microscope (Zeiss, Göttingen, Germany) equipped with three separate bandpass filters (DAPI bandpass, Green single bandpass, Red single bandpass) and a high sensitive monochrome charge coupled device camera (Photometrics, Tuscon, AZ). For each analysis, the averaged chromosome-specific green to red fluorescence ratios and their $95 \%$ confidence intervals (CI) from at least ten well-selected metaphases were plotted using the Quips CGH software (distributed through Applied Imaging, Newcastle, UK). Relative copy number changes were interpreted as gains when the average greento-red ratio exceeded 1.2 or 2 (high-level amplification), and

Table 1 Antibodies used in the study

\begin{tabular}{lllll}
\hline Antibody specificity & Source & Type & Dilution & Pretreatment \\
\hline Vimentin & Novocastra (Nunningen, Switzerland) & $\mathrm{mm}$ & $1: 50$ & Microwave \\
CD15 & Dako Cytomation (Baar, Switzerland) & $\mathrm{mm}$ & $1: 100$ & Microwave \\
CD30 & Dako Cytomation (Baar, Switzerland) & $\mathrm{mm}$ & Prediluted & Microwave \\
CD34 & Dako Cytomation (Baar, Switzerland) & $\mathrm{mm}$ & $1: 200$ & Microwave \\
CD68 & Dako Cytomation (Baar, Switzerland) & $\mathrm{mm}$ & $1: 1,600$ & Microwave \\
CK22 & Biomeda (Foster City, USA) & $\mathrm{mm}$ & $1: 200$ & Microwave \\
EMA & Dako Cytomation (Baar, Switzerland) & $\mathrm{mm}$ & $1: 1600$ & Microwave \\
Desmin & Dako Cytomation (Baar, Switzerland) & $\mathrm{mm}$ & $1: 4,000$ & Microwave \\
Actin & NeoMarkers (Fremont, USA) & $\mathrm{mm}$ & $1: 25$ & Microwave \\
SMA & Dako Cytomation (Baar, Switzerland) & $\mathrm{mm}$ & Prediluted & None \\
S100 Protein & Medite (Nunningen, Switzerland) & $\mathrm{rp}$ & $1: 1,600$ & Microwave
\end{tabular}

$C D$ Cluster of differentiation, $C K$ cytokeratin, $E M A$ epithelial membrane antigen, $S M A$ smooth muscle actin, $m m$ mouse monoclonal, $r p$ rabbit polyclonal 
as losses when the corresponding ratio was less than 0.8 . Exceptionally, in cases with only trends not reaching the aforementioned thresholds, deviations from normal were classified as gains or losses when the $95 \%$ CI varied beyond the ratio of 1.0. An event was defined as a gain or a loss of (part of) a chromosomal arm. Chromosomal regions 1p32-pter, 13p, $14 \mathrm{p}, 15 \mathrm{p}, 19,21 \mathrm{p}, 22 \mathrm{p}$, telomeres and constitutive heterochromatic regions at 1q, 9q, 16q, and Yq reported to produce false results by CGH were excluded from all analyses [7].

\section{DNA image cytometry}

The specimens were evaluated with the semiautomatic AUTOCYTETM cell analytical system (Carl Zeiss AG, CH 8706 Feldmeilen) using a Zeiss AxioplanTM microscope. On average, 135 (104-153) of the most abnormally looking tumor cells were selected as test cells, whereas 50 nuclei of lymphocytes were measured as internal standard.

The histograms were analyzed following the recommendations of the European Society for Analytical and Cellular Pathology (ESACP) [3, 4]. Both stemlines and single events were considered. The key data of DNA cytometry, i.e., integrated optical density and nuclear area, were transferred to the server EUROQUANTTM (http://euroquant.med. tu-dresden.de) at the Institute of Pathology, Technical University/Dresden, which is available free of charge to any user for quality assurance [5]. There, the data were standardized and evaluated on the basis of statistic models, by which, the number of stemlines, their position on the ploidy axis, and their coefficient of variation are determined. EUROQUANT delivers the modal DNA values of peaks and the coefficient of variation $(\mathrm{CV})$ value of stemlines and calculates several quality parameters defined by the ESACP (e.g., parameters for a control of measured glare factors and of the homogeneity of measurement) and compares them to established limit values. Single events were directly analyzed on the basis of the AUTOCYTE data. Ploidy classes were defined as follows: euploid/ diploid: 1.8-2.2 c; tetraploid/polyploid: 3.6-4.4 c; octaploid: 7.2-8.8 c; aneuploid: stemline outside of a euploid region ( $2 \mathrm{c}, 4 \mathrm{c}, 8 \mathrm{c}$, etc.), single events $>8.8 \mathrm{c}$.

\section{Results}

\section{Clinical findings}

Clinical features of the two cases are summarized in Table 2. One patient noticed a slowly growing painless mass in the right index finger and was treated surgically by local excision (case 1). The other patient suffered from pain in the right popliteal fossa and observed a slowly growing tumor that turned out to be located inside the tibial nerve (case 2). After histologic diagnosis, treatment consisted in isolated limb perfusion with tumor necrosis factor alpha $(\mathrm{TNF}-\alpha)$ and Melphalan followed by neoadjuvant percutaneous radiotherapy and local excision without destruction of major fascicles. On histologic examination, no viable tumor was detectable anymore, and the patient did not sustain sensory or motor deficits (Fig. 1). At initial surgery, both tumors showed a myxoid aspect with no signs of necrosis or hemorrhage on cut section. Patient 1 developed local recurrence 5 months after initial resection and underwent re-excision followed by another recurrence 16 months later. The second patient is still free of disease (follow-up 14 months).

\section{Microscopical and immunohistochemical findings}

Light microscopy showed cell-rich tumors containing myxoid and hyaline areas and zones of fibrosis (Fig. 2). Tumor cells revealed pronounced nuclear polymorphism, including differences in nuclear size and form. Some cells were rather small and spindle-shaped, while others revealed big vesicular nuclei and prominent nucleoli, focally resembling ganglion cells or virocytes (Fig. 2f). Additionally, multinucleated cells and lipoblast-like cells with multiple intracytoplasmatic mucin-containing vacuoles were present in all cases (Fig. 2a-c). The inflammatory infiltrates were dense and particularly prominent in the fibrotic and myxoid areas of the tumors. Immunohistochemically all tumors were strongly positive for vimentin and focally positive for CD68 (not shown). Expression of CK22, CD15, CD30, epithelial membrane antigen (EMA), S100 protein, or desmin could not be detected in any of the two cases.

Table 2 Clinical features

\begin{tabular}{lllll}
\hline Case & Sex/age & Location & Size & Follow-up \\
\hline 1 & F/51 & Right index finger & $2 \times 1.5 \times 1 \mathrm{~cm}$ & Recurrences after 5 and 16 months \\
2 & F/75 & Right popliteal fossa, tibial nerve & $4 \times 2 \times 1.7 \mathrm{~cm}$ & 14 months NED \\
\hline
\end{tabular}

$N E D$ No evidence of disease, $M$ male, $F$ female 
Fig. 1 a Case 2: T1-weighted MR showing a hypointense tumor surrounding the tibial nerve (yellow arrows). b Case 2 : Intraoperative situation with isolated nerve before tumor resection (yellow arrows)
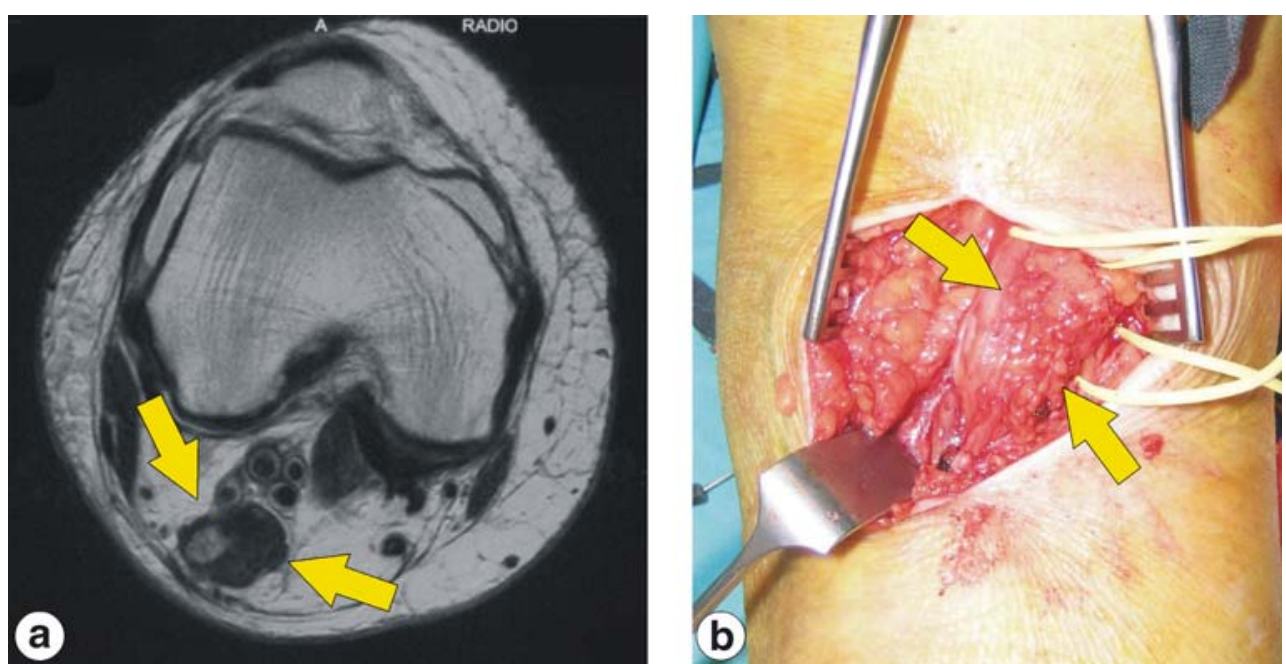

Fig. 2 a Case $1-\mathrm{HE} \times 400$ multivacuolated lipoblast-like tumor cells admixed with a loose inflammatory infiltrate. b Case $1-\mathrm{HE} \times 400$ : tumor cells are embedded in a myxohyaline stroma. c Case $1-\mathrm{HE} \times 640$ : lipoblast-like tumor cells in close contact to lymphocytes and some eosinophils. d Case 2-HE $\times 200$ : spindleshaped tumor cells admixed with a dense inflammatory infiltrate and embedded in a collagenous hyalinized stroma e Case 2-HE $\times 200$ : spindleshaped tumor cells and lymphocytes in a myxoid stroma. $\mathbf{f}$ Case 2-HE $\times 640$ : Ganglion-like tumor cells with large eosinophilic nucleoli accompanied by lymphocytes and plasma cells
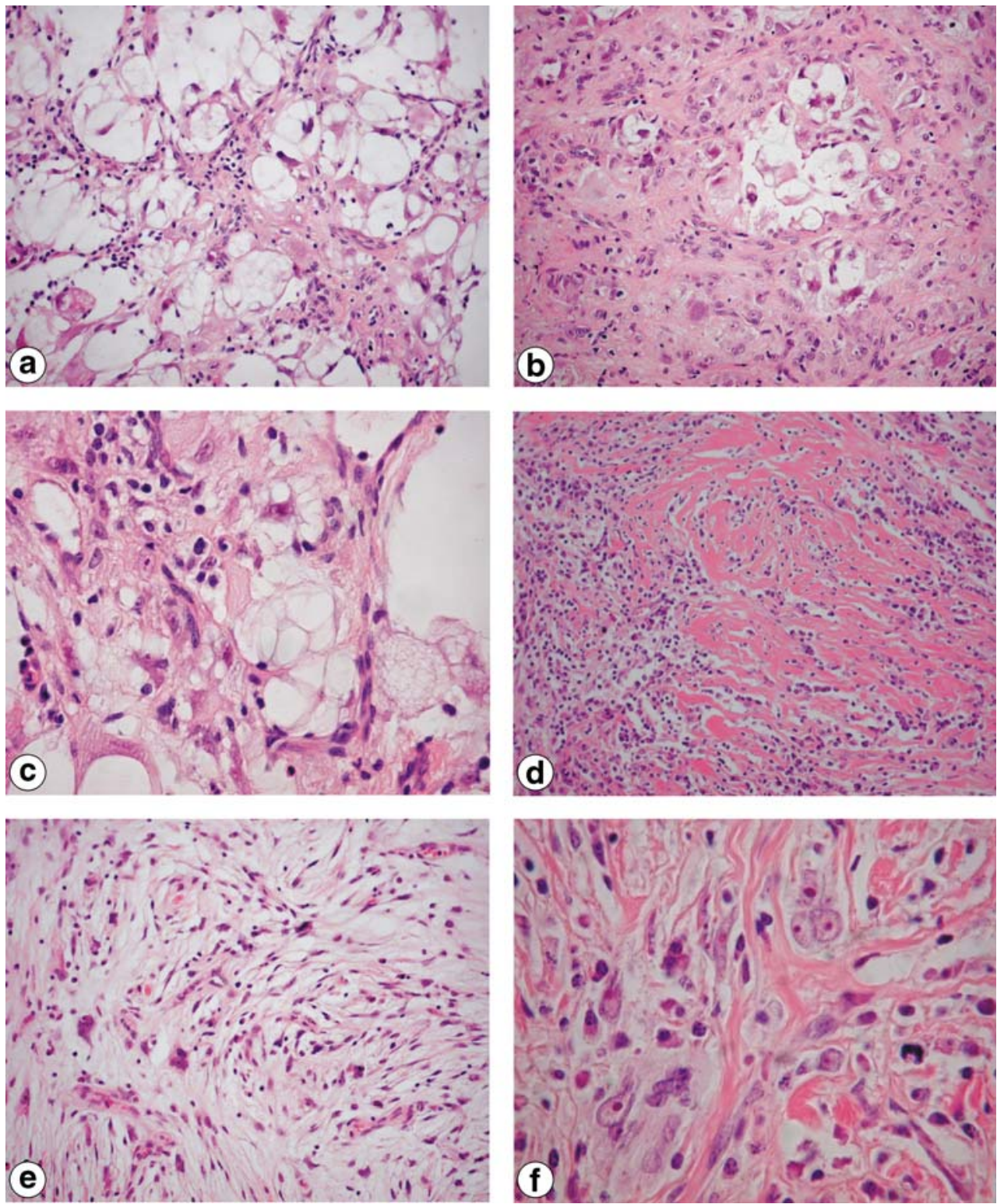
Table 3 CGH analysis

\begin{tabular}{ll} 
Case & Results \\
\hline 1 & $0.20:$ rev ish enh(3p11p12,7q31q32) \\
& trend enh(1p, 18), dim(6q14qter) \\
2 & 0,20: rev ish enh(5pterq32, 7p15p21, 14q12q21)
\end{tabular}

Comparative genomic hybridization

DNA copy number changes were identified in both cases (Table 3, Fig. 3). The number of changes ranged from two (case 1) to three (case 2). There were no specific common gains or losses, but both investigated cases showed gains at chromosome 7.

Fig. 3 a-b Comparative genomic analyses of cases $1-2$
DNA image cytometry

All tumors revealed aneuploid stemlines and additional aneuploid single events. Modal DNA value of peaks ranged between $2.54 \mathrm{c}$ and $2.72 \mathrm{c}$ (mean $2.63 \mathrm{c}$ ). The $\mathrm{CV}$ varied between 7.31 and $7.36 \%$ (mean $7.34 \%$ ).

\section{Discussion}

Myxoinflammatory fibroblastic sarcomas are rare low-grade tumors typically presenting as painless and slowly growing masses of the distal extremities. According to the Medline database, 133 cases have been reported up to now, including a
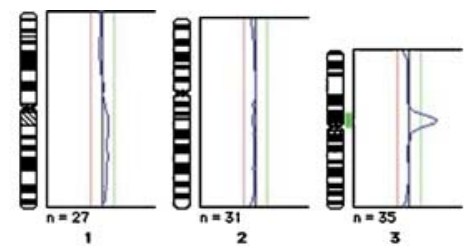

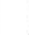
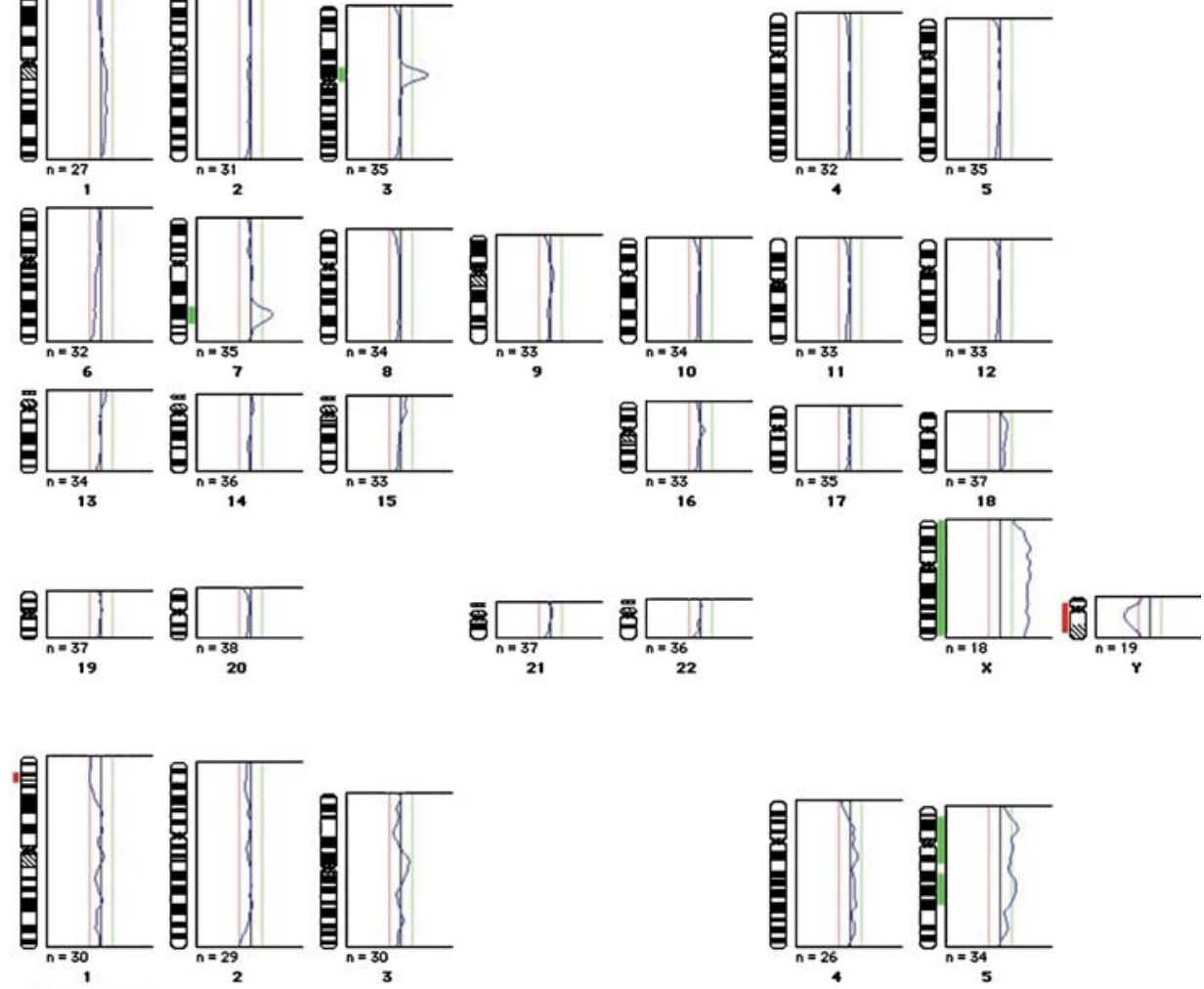

b
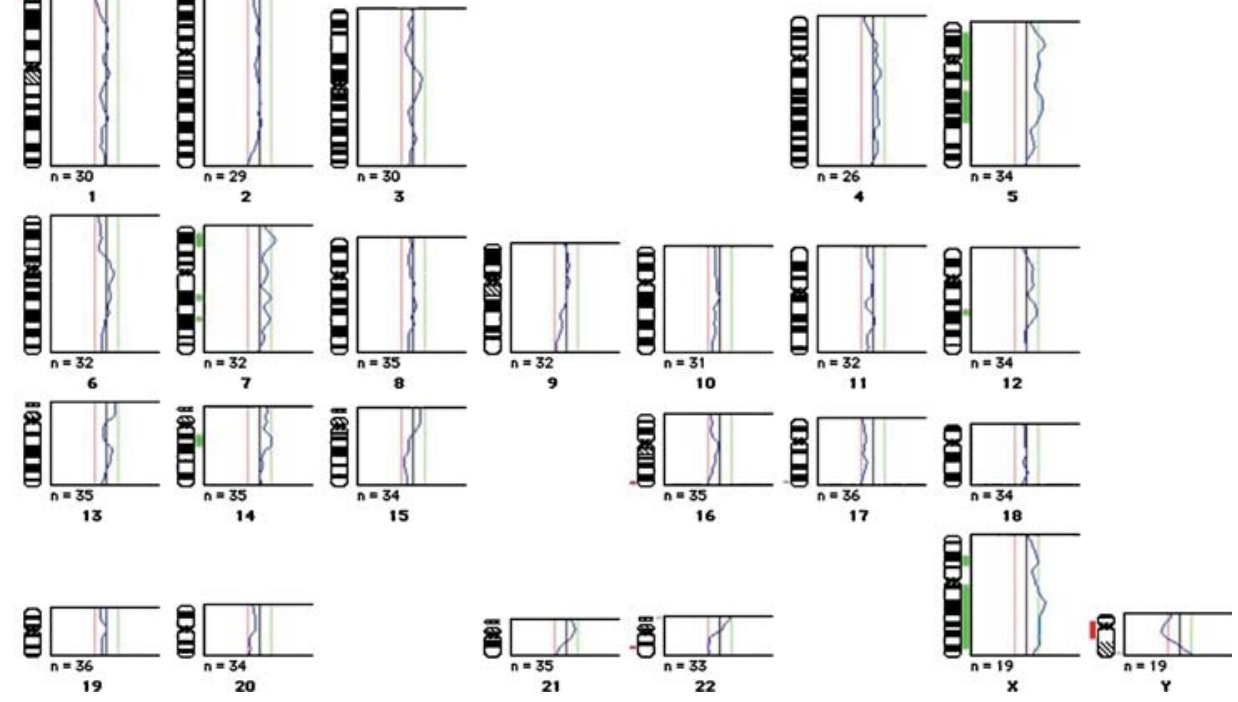
several tumors of the proximal extremities as well (upper arm, thigh) [1, 6, 8-10, 12-17]. Both investigated cases revealed the characteristic histomorphological and immunohistochemical pattern of MIFS. Besides the alternating and confluencing myxoid and hyaline stroma containing a predominantly lymphoplasmocytic inflammatory infiltrate, all tumors exhibited intermixed spindle cells, large polygonal and partially bizarre ganglion-like cells with huge inclusion-like nucleoli and bubbly multinucleated cells resembling lipoblasts (Fig. 2). Immunohistochemically, the tumor cells stained positive for Vimentin and CD68.

Cytogenetic data on chromosomal aberrations on MIFS are limited. To our knowledge, only two cases have been investigated by conventional cytogenetic and fluorescence in situ hybridization analyses so far. One case showed a derivate chromosome 13 with additional material on the short arm and supernumerary ring chromosomes, while the other exhibited a complex karyotype with a reciprocal translocation $\mathrm{t}(1 ; 10)$ (p22;q24) and losses of chromosomes 3 and 13 [9, 11]. The two cases of the present study also revealed aberrant karyotypes involving gains at several different chromosomal regions. Although no specific common aberration was observed, both cases shared gains at chromosome 7. Losses of chromosomal regions were not detected. Furthermore, no chromosomal alterations were shared with the previously investigated two MIFS [9, 11]. DNA image cytometry revealed both tumors of our series to be DNA aneuploid, which has never been investigated so far.

In conclusion, the two MIFS of the present study show considerable genetic diversity, which is in keeping with the two previously investigated cases. However, no single marker or profile of DNA copy number changes characterizing all tumors could be detected. Further, cytogenetic or $\mathrm{CGH}$ analyses have to be awaited to define specific cytogenetic profiles of MIFS and possible separate subtypes of MIFS, respectively.

\section{References}

1. Ebhardt H, Kosmehl H, Katenkamp D (2001) Acral myxoinflammatory fibroblastic sarcoma. Six cases of a tumor entity. Pathologe 22:157-161

2. Fletcher C, Unni K, Mertens F (2002) World Health Organization classification of tumours: pathology and genetics of tumours of soft tissue and bone. IARC, Lyon, France
3. Giroud F, Haroske G, Reith A, Bocking A (1998) 1997 ESACP consensus report on diagnostic DNA image cytometry. Part II: specific recommendations for quality assurance. European Society for Analytical Cellular Pathology. Anal Cell Pathol 17:201-208

4. Haroske G, Giroud F, Reith A, Bocking A (1998) 1997 ESACP consensus report on diagnostic DNA image cytometry. Part I: basic considerations and recommendations for preparation, measurement and interpretation. European Society for Analytical Cellular Pathology. Anal Cell Pathol 17:189-200

5. Haroske G, Meyer W, Theissig F, Schubert K, Kunze KD (1998) Remote quantitation server for quality assurance in DNA ploidy analysis. Anal Quant Cytol Histol 20:302-312

6. Jurcic V, Zidar A, Montiel MD, Frkovic-Grazio S, Nayler SJ, Cooper K et al (2002) Myxoinflammatory fibroblastic sarcoma: a tumor not restricted to acral sites. Ann Diagn Pathol 6:272-280

7. Kallioniemi OP, Kallioniemi A, Piper J, Isola J, Waldman FM, Gray JW et al (1994) Optimizing comparative genomic hybridization for analysis of DNA sequence copy number changes in solid tumors. Genes Chromosomes Cancer 10:231-243

8. Kinkor Z, Mukensnabl P, Michal M (2002) Inflammatory myxohyaline tumor with massive emperipolesis. Pathol Res Pract 198:639-642

9. Lambert I, Debiec-Rychter M, Guelinckx P, Hagemeijer A, Sciot R (2001) Acral myxoinflammatory fibroblastic sarcoma with unique clonal chromosomal changes. Virchows Arch 438:509512

10. Lang JE, Dodd L, Martinez S, Brigman BE (2006) Case reports: acral myxoinflammatory fibroblastic sarcoma: a report of five cases and literature review. Clin Orthop Relat Res 445:254-260

11. Mansoor A, Fidda N, Himoe E, Payne M, Lawce H, Magenis RE (2004) Myxoinflammatory fibroblastic sarcoma with complex supernumerary ring chromosomes composed of chromosome 3 segments. Cancer Genet Cytogenet 152:61-65

12. Meis-Kindblom JM, Kindblom LG (1998) Acral myxoinflammatory fibroblastic sarcoma: a low-grade tumor of the hands and feet. Am J Surg Pathol 22:911-924

13. Michal M (1998) Inflammatory myxoid tumor of the soft parts with bizarre giant cells. Pathol Res Pract 194:529-533

14. Montgomery EA, Devaney KO, Giordano TJ, Weiss SW (1998) Inflammatory myxohyaline tumor of distal extremities with virocyte or Reed-Sternberg-like cells: a distinctive lesion with features simulating inflammatory conditions, Hodgkin's disease, and various sarcomas. Mod Pathol 11:384-391

15. Oda Y, Tamiya S, Oshiro Y, Hachitanda Y, Kinukawa N, Iwamoto $Y$ et al (2002) Reassessment and clinicopathological prognostic factors of malignant fibrous histiocytoma of soft parts. Pathol Int 52:595-606

16. Pohar-Marinsek Z, Flezar M, Lamovec J (2003) Acral myxoinflammatory fibroblastic sarcoma in FNAB samples: can we distinguish it from other myxoid lesions? Cytopathology 14:73-78

17. Sakaki M, Hirokawa M, Wakatsuki S, Sano T, Endo K, Fujii Y et al (2003) Acral myxoinflammatory fibroblastic sarcoma: a report of five cases and review of the literature. Virchows Arch 442:25-30

18. Weiss SW, Goldblum JR (2001) Enzinger and Weiss's soft tissue tumors. Mosby, St. Louis, Missouri, USA 